\title{
LUCIFER: Neutrinoless Double Beta Decay search with scintillating bolometers
}

\author{
Gabriele Piperno* \\ Dipertimento di Fisica - Sapienza, Università di Roma, I-00185 Roma, Italy \\ E-mail: gabriele.piperno@romal.infn.it
}

The Neutrinoless Double Beta Decay (0vDBD) is a powerful tool to test physics beyond the Standard Model and to get insights on the Majorana neutrino nature and mass. Bolometers are excellent detectors to search for this rare decay, thanks to their good energy resolution and to the low background conditions in which they can operate. The current challenge consists in the reduction of the background, represented by environmental $\gamma_{\mathrm{s}}$ and $\alpha \mathrm{s}$, in view of a zero background experiment. This can be obtained with the approach of the LUCIFER project, funded by an European grant, which is based the double read-out of the heat and scintillation light produced by $\mathrm{ZnSe}$ scintillating bolometers, that allows to discriminate between $\beta / \gamma$ and $\alpha$ particles. The LUCIFER experiment aims at a background of the order of $10^{-3}$ counts $/ \mathrm{keV} / \mathrm{kg} / \mathrm{y}$ in the energy region of the $0 v \mathrm{DBD}$ of ${ }^{82} \mathrm{Se}$, an order of magnitude lower with respect to the present generation experiments. Such a low background level will provide a sensitivity on the effective neutrino mass of the order of $100 \mathrm{meV}$. We describe the current status of the LUCIFER project, including results of the recent $R \& D$ activity.

Technology and Instrumentation in Particle Physics 2014,

2-6 June, 2014

Amsterdam, the Netherlands

\footnotetext{
*Speaker.
} 


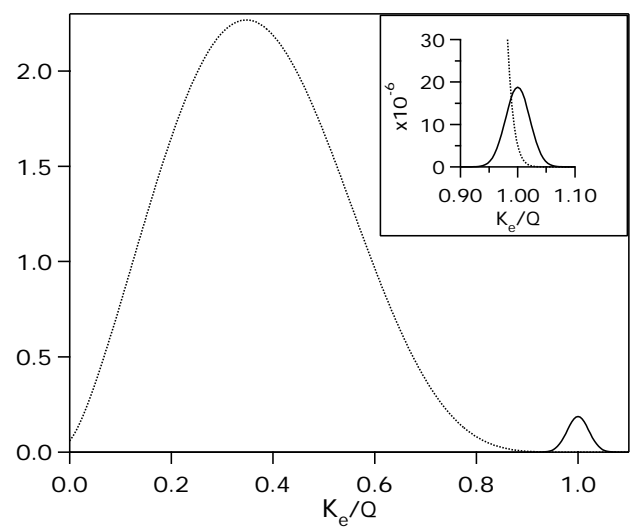

Figure 1: $2 v \mathrm{DBD}$ and $0 v \mathrm{DBD}$ two electrons sum energy spectra. $2 v \mathrm{DBD}$ is a continuum between 0 and $Q_{\beta \beta}$, while $0 v \mathrm{DBD}$ is a peak centered at $Q_{\beta \beta}$. In the inset is shown that a good energy resolution is needed to disentangle the two spectra. The y-axis scale and the $0 v \mathrm{DBD}$ relative peak height are arbitrary.

\section{Neutrinoless Double Beta Decay}

In contemporary physics the neutrino's importance increased considerably during recent years. This is because some of its fundamental properties, nature (Dirac or Majorana) and mass (absolute scale and hierarchy) are still unknown. Even more, the neutrino provides a unique tool to search for signals of physics beyond the Standard Model (SM). In fact the neutrino could be a Majorana particle, differently from all other fermions that are Dirac particles, implying that neutrino and antineutrino are the same. The only way to probe the neutrino nature is to search for the neutrinoless double beta decay.

The "simple" double beta decay with neutrino emission $(2 v$ DBD) is the rarest nuclear decay ever observed in nature [1], in which two neutrons decay into two protons, two electrons and two antineutrinos. This is a second order weak transition allowed by the Standard Model (SM), with lifetimes ranging from $10^{18}$ to $10^{22}$ years [1].

Neutrinoless double beta decay $(0 v \mathrm{DBD})$ is a hypothetical (never observed) double beta decay in which no neutrinos are emitted. $0 v \mathrm{DBD}$ is SM forbidden and can occur only if the neutrino is a Majorana particle and would violate by 2 the lepton number conservation. The importance of this decay is increased by the fact that it depends on the neutrino Majorana mass, so its observation would give an answer to the absolute mass scale question too. For this process there are no measured lifetimes, but only lower limits of the order of $10^{25} \mathrm{y}[2]$.

The experimental signature of the $0 v \mathrm{DBD}$ is extremely simple: two electron of same energy emitted back-to-back, with a total energy equal to the Q-value of the reaction: $Q_{\beta \beta}=M_{m}-M_{d}-$ $2 m_{e}$. Looking at the two electrons sum energy spectra of the two decay, $2 v$ DBD presents a continuous distribution from 0 to the Q-value, while $0 v \mathrm{DBD}$ presents a single peak at the Q-value, as shown in Fig.1.

Considering the very high lifetimes, it is clear that to have chances to see the $0 v \mathrm{DBD}$ peak it is necessary to have a very low background in the region of interest (ROI) and a detector mass of the order $100 \sim 1000 \mathrm{~kg}$, to have a large number of $\beta \beta$ emitter. Looking at Fig.1, in particular the inset, 
one can understand that another requirement to fulfill is a good energy resolution to discriminate the $0 v \mathrm{DBD}$ peak from the right tail of the $2 v \mathrm{DBD}$ distribution.

In a $0 v$ DBD experiment the sensitivity is defined as the half-life corresponding to a signal that can be emulated by a background fluctuation at a certain confidence level, given in Gaussian standard deviation $\left(n_{\sigma}\right)$ :

$$
S^{b k g}=\frac{\ln 2}{n_{\sigma}} N_{a} \varepsilon \frac{\rho \eta}{\mathbf{A}} \sqrt{\frac{M t}{B \Delta E}}
$$

where $N_{a}$ the Avogadro number, $\varepsilon$ is the detection efficiency, $\rho$ the stoichiometric coefficient, $\eta$ the isotopic abundance of the $\beta \beta$ emitter, $\mathbf{A}$ the atomic mass number, $M$ the detector mass in $\mathrm{kg}$, $t$ the experiment livetime in $\mathrm{y}, B$ the background in counts $/ \mathrm{keV} / \mathrm{kg} / \mathrm{y}, \Delta E$ the energy resolution in $\mathrm{keV}$ and the resultant value of $S^{b k g}$ is expressed in y. The most significant parameters are the ones under the square root: $M, t, B$ and $\Delta E$. From the experimental point of view some limitations arise on these parameters: $M$ could not be larger than some tons, $t$ generally is supposed to be in the interval $5 \sim 10 \mathrm{y}$ and $\Delta E$ is somehow fixed once the detection technique is selected. Then $B$ is the most important parameter to work with to improve the sensitivity. In addition, if the background is low enough to reach the zero background limit, the sensitivity formula changes:

$$
S^{0 b k g}=-\frac{\ln 2}{\ln (1-\text { C.L. } / 100)} N_{a} \varepsilon \frac{\rho \eta}{\mathbf{A}} M t
$$

becoming linear with $M$ and $t$.

\section{The LUCIFER experiment}

\subsection{Isotope selection}

In nature there are a ten of isotopes that undergo DBD. To select the good one for an experiment, the Q-value of the reaction and the isotopic abundance of the atom must be taken into account (see Table 1). From an experimental point of view is better to select an isotope with $Q_{\beta \beta}>2615 \mathrm{keV}$, since above this energy (a ${ }^{208} \mathrm{Tl} \gamma$ line) the $\gamma$ radioactivity is reduced by a factor $\sim 100$. Adding the information of the isotopic abundance (see Tab.1), ${ }^{82} \mathrm{Se}\left(Q_{\beta \beta}=2997 \mathrm{keV}\right.$, $\eta=8.7 \%)$ and ${ }^{100} \mathrm{Mo}\left(Q_{\beta \beta}=3034 \mathrm{keV}, \eta=9.6 \%\right)$ seem to be a good choices. These isotopes show other good features, like the possibility to grow crystals with a large mass fraction of these elements and the possibility to follow the enrichment way to increase the $\beta \beta$ emitter number.

\subsection{Scintillating bolometers}

Among the various possible detection technique [4], the bolometric one is particularly indicated to search for $0 v \mathrm{DBD}$. A bolometer is a crystal operated as a calorimeter at a temperature of $\simeq 10 \mathrm{mK}$. In this, an energy release by a particle interaction raises the crystal temperature $(\sim 0.1 \mathrm{mK} / \mathrm{MeV})$. This temperature variation is measured by means of a thermometer, in our case a neutron transmutation doped (NTD) Ge thermistor. Then the accumulated heat is dissipated with a coupling to a thermal bath (Fig.2 left for scintillating bolometer schema, see later). Each event in 


\begin{tabular}{|c|c|c|}
\hline Isotope & $\begin{array}{c}Q_{\beta \beta} \\
{[\mathrm{keV}]}\end{array}$ & $\begin{array}{c}\text { Iso. Abb. } \\
{[\%]}\end{array}$ \\
\hline \hline${ }^{48} \mathrm{Ca}$ & 4274 & 0.187 \\
${ }^{76} \mathrm{Ge}$ & 2039 & 7.4 \\
${ }^{82} \mathrm{Se}$ & 2997 & 8.7 \\
${ }^{100} \mathrm{Mo}$ & 3034 & 9.6 \\
${ }^{116} \mathrm{Cd}$ & 2814 & 7.5 \\
${ }^{130} \mathrm{Te}$ & 2528 & 33.8 \\
${ }^{136} \mathrm{Xe}$ & 2458 & 8.9 \\
${ }^{150} \mathrm{Nd}$ & 3368 & 5.6 \\
\hline
\end{tabular}

Table 1: Q-values and isotopic abundances of the principal candidates for a $0 v \beta \beta$ experiment. Numbers from [3].

a bolometer correspond to a registered pulse (shape is shown in Fig.2 right) which height depends on the energy released.

The reasons why the bolometric technique is very suitable are different:

- crystals can be grown with $\beta \beta$ emitter directly inside: high detection efficiency $(75 \div 80 \%)$,

- energy resolution of order of per mille,

- high radio-purity is reachable,

- scalability is quite simple,

- different compounds can be used to study different isotope (necessary for a convincing $0 v$ DBD observation).

To reduce as much as possible all the background sources many precautions are taken: cosmic radiation is reduced placing the experiment in underground laboratories, the laboratories environmental radioactivity by using shielding, the shielding radioactivity with an accurate material selection and cleaning and the bolometers contaminations by means of a radio-pure crystals growth.

The bolometric experiment CUORE-0 was build paying attention to all these points and in particular to validate the steps of the detector construction adopted to reduce the background of the future experiment CUORE [5]: crystals production method, cleaning procedure of the materials and tower assembly line. With these precautions CUORE-0 reached a background of $2 \times 10^{-2}$ counts $/ \mathrm{keV} / \mathrm{kg} / \mathrm{y}$ in the LUCIFER ROI [6]. It has been demonstrated that the principal source of background for these experiments consists of smeared $\alpha$ particles located on the crystal surface or on the tower material facing the crystal, that do not release all their energy in the bolometer, but only a random fraction of it $[7,8]$. To reach the zero background limit for LUCIFER of $10^{-3}$ counts $/ \mathrm{keV} / \mathrm{kg} / \mathrm{y}$, a more effective background reduction is needed.

A solution to this problem comes from the scintillating bolometers. In this case less than $1 \%$ of the total energy is converted into scintillation photons and, since there is a different light yield for $\alpha$ and $\beta / \gamma$, it is possible to identify and reject the $\alpha$ background [9]. Due to the working conditions the best choice for a light detector is to use a second bolometer, generally a Ge disk 

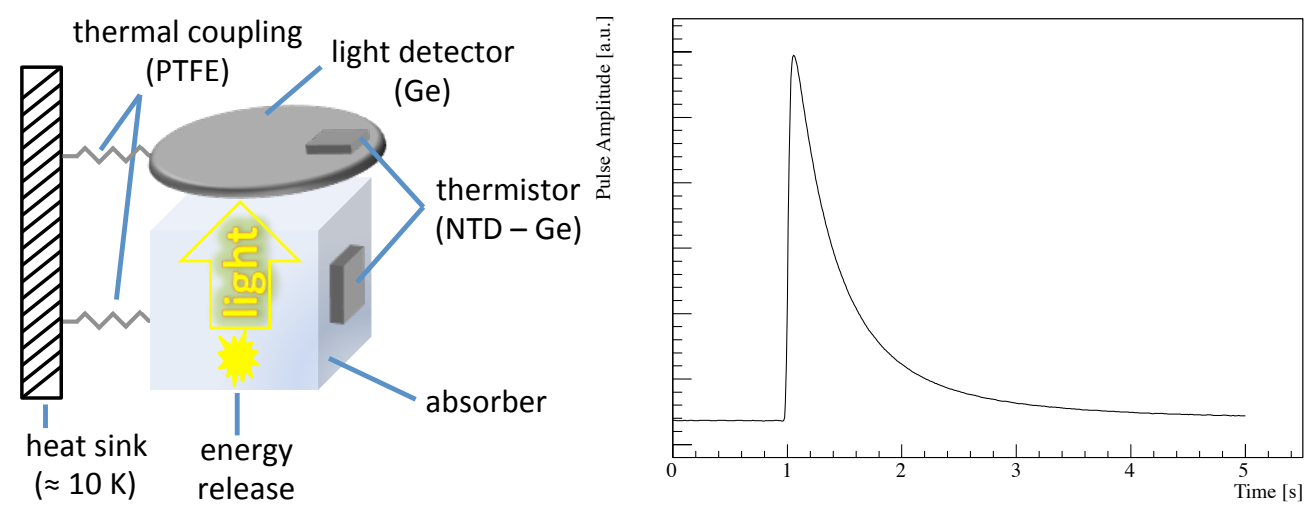

Figure 2: Left: schema of (scintillating) bolometer operation. An energy release by a particle interaction raises the crystal temperature. Then this temperature variation is measured by a thermometer, a neutron transmutation doped (NTD) Ge thermistor. The accumulated heat is subsequently dissipated by means of a coupling to a thermal bath. In a scintillating bolometer less than $1 \%$ of the total energy goes into scintillation photons. To read the this light and discriminate the $\alpha$ background, a second bolometer (generally a Ge disk) is operated as light detector. Right: an event in a bolometer is registered as a pulse, which heigh depends on the energy release.

\begin{tabular}{|c|c|c|c|c|c|}
\hline$\beta \beta$ emitter & crystal & $\begin{array}{c}Q_{\beta \beta} \\
{[\mathrm{keV}]}\end{array}$ & $\begin{array}{c}\beta \beta \text { emitter mass } \\
{[\mathrm{kg}]}\end{array}$ & $Q F$ & $\begin{array}{c}\text { FWHM at } Q_{\beta \beta} \\
{[\mathrm{keV}]}\end{array}$ \\
\hline \hline${ }^{82} \mathrm{Se}$ & $\mathrm{ZnSe}^{100} \mathrm{Mo}$ & 2997 & $\approx 9.8$ & 4.2 & 16.5 \\
$\mathrm{ZnMoO}_{4}$ & 3034 & $\approx 6.2$ & 0.14 & 7 \\
\hline
\end{tabular}

Table 2: Principal characteristics of two samples of the two alternatives for LUCIFER. ZnSe shows a $Q F=4.2$, while smaller than 1 was expected. The FWHM at $Q_{\beta \beta}$ for $\mathrm{ZnSe}$ is evaluated using the correlation between the light and the heat channels (while for $\mathrm{ZnMoO}_{4}$ the resolution remains the same also using this approach). $Q F$ and FWHM may vary from sample to sample.

of $\approx 5 \mathrm{~cm}$ diameter and $1 \mathrm{~mm}$ of maximum thickness [10,11]. Using the light detector one can produce a scatter plot with on the x-axis the energy of the event and on the y-axis the detected light. Starting from this it is possible to define a quantity, called Quenching Factor, that quantifies the separation between the $\alpha$ and the $\beta / \gamma$ band: $Q F=\frac{\alpha_{\text {detectedlight }}}{\beta / \gamma_{\text {detectedlight }}}$ for events of the same energy and is expected to be smaller than one.

\subsection{Detector layout}

LUCIFER (Low background Underground Installation For Elusive Rates) will search for $0 v \beta \beta$ of ${ }^{82} \mathrm{Se}$ with $\mathrm{ZnSe}$ scintillating bolometer or, alternatively, the $0 v \beta \beta$ of ${ }^{100} \mathrm{Mo}$ with $\mathrm{ZnMoO}_{4}$ scintillating bolometers. It will be hosted in the underground Laboratori Nazionali del Gran Sasso ( $3650 \mathrm{~m}$. w. e.) near L'Aquila, Italy. The detector will consists of 36 cylindrical crystals $(45 \mathrm{~mm}$ diameter, $55 \mathrm{~mm}$ height), enriched at $95 \%$, each one equipped with a Ge disk ( $44 \mathrm{~mm}$ diameter, $180 \mu \mathrm{m}$ thickness) operated as a light detector. It will be arranged in a tower of 9 floors with 4 crystals per floor. In Table 2 the fundamental characteristics of two samples of the two possible choice for the experiment are summarized. 

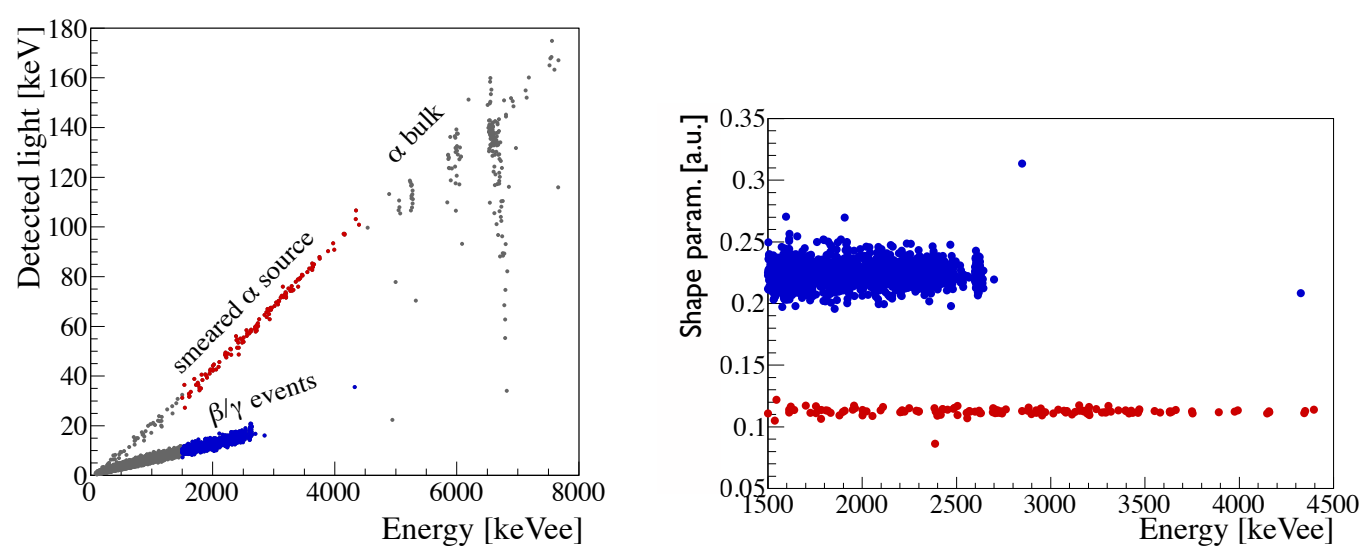

Figure 3: Left: scatter plot light vs heat obtained for the $\mathrm{ZnSe}$ bolometer. This compound is the unique known to show a $Q F>1$. Right: using a shape parameter from the light detector it is possible to discriminate $\alpha$ background in case the light from $\mathrm{ZnSe}$ is not correctly detected and an $\alpha$ event falls in the $\beta / \gamma$ region. Images from [12].

\subsubsection{ZnSe scintillating bolometers}

We tested many ZnSe bolometers, with different physical and bolometric properties. The one presented here is a $430 \mathrm{~g}$ crystal (see Table 2 for properties), that shows an excellent particle identification. As can be seen in Fig.3 left, this ZnSe, like all the other we tested, shows a peculiar $Q F$ larger than 1. At the moment this is the unique known bolometer that has this unexpected feature. This characteristics can become a problem: if the light is not correctly detected, an $\alpha$ particle could fall in the $\beta / \gamma$ region of the scatter plot. To solve this problem it is possible to use the information that comes from the shape of the pulse in the light detector, as visible in Fig. 3 right. Here a shape parameter, based on the difference between every single pulse and the average one, is shown.

The measurement of this bolometer tells that an effective $\alpha$ background rejection is achievable and that the region where $0 v \beta \beta$ of ${ }^{82} \mathrm{Se}$ is expected to be has no events in $524 \mathrm{~h}$ of background measurement.

Crystal contaminations are $3 \times 10^{-4}$ counts $/ \mathrm{keV} / \mathrm{kg} / \mathrm{y}$ in ${ }^{238} \mathrm{U}$ and $3 \times 10^{-3}$ counts $/ \mathrm{keV} / \mathrm{kg} / \mathrm{y}$ in ${ }^{232} \mathrm{Th}$, while an external background of the order of $10^{-3}$ counts $/ \mathrm{keV} / \mathrm{kg} / \mathrm{y}$ is reliable. This leads, for a $5 \mathrm{y}$ measurement of $\mathrm{a} \approx 20 \mathrm{~kg}$ detector, to expect 1 count of background in the ROI, meaning that a zero background detector is attainable.

\subsection{2 $\mathrm{ZnMoO}_{4}$ scintillating bolometers}

Several $\mathrm{ZnMoO}_{4}$ crystals were measured in our facility, with masses ranging from 30 to $330 \mathrm{~g}$. The results presented here are from the largest one, which fundamental parameters are in Table 2. This bolometer features a very good particle identification, as shown in Fig. 4 left, given the very low $Q F$ and very narrow bandwidth of $\alpha$ and $\beta / \gamma$ populations. The energy resolution of $7 \mathrm{keV}$ at the Q-value is an excellent result, like the very low Contaminations (measured in a $524 \mathrm{~h}$ run): $<10 \mu \mathrm{Bq} / \mathrm{kg}$ in ${ }^{238} \mathrm{U}$ and ${ }^{232} \mathrm{Th}$, that lead, also in this case, to a background free detector. In particular the $\beta / \gamma$ region of the scatter plot, where the ${ }^{100} \mathrm{Mo} 0 v \beta \beta$ should fall, is empty of events. 

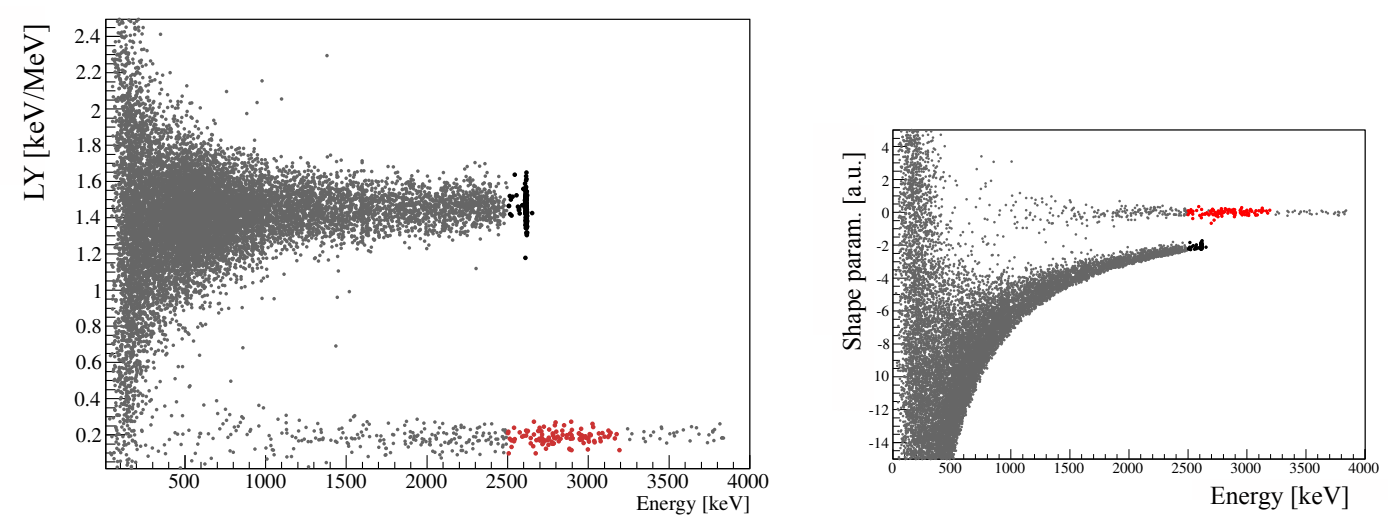

Figure 4: Left: with a $Q F=0.14$ and narrow bandwidths, $\alpha$ and $\beta / \gamma$ particles are well separated in the $\mathrm{ZnMoO}_{4}$ bolometer. Right: this bolometer is capable to discriminate $\alpha$ and $\beta / \gamma$ interactions using a shape parameter directly from its own pulses. Images from [13].

\begin{tabular}{|c|c|c|c|}
\hline crystal & $\begin{array}{c}\text { live time } \\
{[\mathrm{y}]}\end{array}$ & $\begin{array}{c}90 \% \text { C.L. } T_{1 / 2}^{0 v} \\
{\left[10^{26} \mathrm{y}\right]}\end{array}$ & $\begin{array}{c}\left\langle m_{\beta \beta}\right\rangle \\
{[\mathrm{meV}]}\end{array}$ \\
\hline \hline $\mathrm{ZnSe}$ & 5 & 0.6 & $65 \div 194$ \\
$\mathrm{ZnSe}$ & 10 & 1.2 & $46 \div 138$ \\
$\mathrm{ZnMoO}_{4}$ & 5 & 0.3 & $60 \div 170$ \\
$\mathrm{ZnMoO}_{4}$ & 10 & 0.6 & $42 \div 120$ \\
\hline
\end{tabular}

Table 3: $90 \%$ C.L. sensitivity $\left(T_{1 / 2}^{0 v}\right)$ for 5 and $10 \mathrm{y}$ of $\mathrm{ZnSe}$ and $\mathrm{ZnMoO}_{4}$ and relative effective neutrino masses $\left(\left\langle m_{\beta \beta}\right\rangle\right)$.

As a last remark, we noticed in this bolometer the pulse shape depends on the interaction nature, meaning that it is possible to discriminate $\alpha$ particles using directly the $\mathrm{ZnMoO}_{4}$ without the help of the light detector (Fig.4 right).

\subsection{Sensitivity}

Considering that a zero background detector is achievable, the sensitivity formula to use is (1.1). Corresponding values $\left(T_{1 / 2}^{0 v}\right)$ for 5 or $10 \mathrm{y}$ of measurement of $\mathrm{ZnSe}$ and $\mathrm{ZnMoO}_{4}$ are listed in Table 3 for a $90 \%$ C.L. These values can be comparative with the $90 \%$ C.L. sensitivity of $5 \mathrm{y}$ of CUORE, an experiment that will have a mass of $741 \mathrm{~kg}: 0.95 \times 10^{26} \mathrm{y}$.

The last column in Table 3 refers to the neutrino effective mass, which is related to the sensitivity by:

$$
\left(T_{1 / 2}^{0 v}\right)^{-1}=G^{0 v}\left|M^{0 v}\right|^{2}\left(\frac{\left\langle m_{\beta \beta}\right\rangle}{m_{e}}\right)^{2}
$$

where $G^{0 v}$ is the phase space factor, $M^{0 v}$ the Nuclear Matrix Element and $m_{e}$ the electron mass. Explorable values of $\left\langle m_{\beta \beta}\right\rangle$ ranges from some ten of $\mathrm{mV}$ to two hundreds of $\mathrm{mV}$. 


\section{Conclusions}

Neutrinos still pose open questions about their mass (hierarchy and absolute scale) and nature (Dirac or Majorana). Neutrinoless double beta decay, if observed, could answer to these questions. Among the various possible techniques to search for this decay, the bolometric one seems to be particularly indicated. Using scintillating bolometers coupled to light detectors it will be possible to achieve the zero background limit, discriminating between $\alpha$ background and $\beta / \gamma$ signal. This allows the experiment to have a sensitivity that increases linearly with experimental livetime and detector mass.

LUCIFER is a next generation experiment demonstrator, that will search for ${ }^{82} \mathrm{Se} 0 v \beta \beta$ with $\mathrm{ZnSe}$ scintillating crystals, or $0 v \beta \beta$ of ${ }^{100} \mathrm{Mo}$ with $\mathrm{ZnMoO}_{4}$ scintillating crystals. Using these compounds for $\mathrm{a} \approx 20 \mathrm{~kg}$ detector in $5 \mathrm{y}$ of measurement in a background free condition, the sensitivity on $0 v \beta \beta$ will be of the order of $10^{26} \mathrm{y}$, corresponding to a neutrino effective mass of the order of $100 \mathrm{meV}$.

\section{References}

[1] A. S. Barabash, Precise half-life values for two-neutrino double-Î́s decay, Phys. Rev. C 81 (3) Article ID 035501.

[2] F. T. Avignone III, S. R. Elliott, and J. Engel, Double beta decay, Majorana neutrinos, and neutrino mass, Rev. Mod. Phys. 80 (2) 481.

[3] D. R. Artusa et al., Exploring the Neutrinoless Double Beta Decay in the Inverted Neutrino Hierarchy with Bolometric Detectors, arXiv:1404.4469.

[4] O. Cremonesi, M. Pavan, Challenges in double beta decay, Adv. High Energy Phys. 2014 Article ID 951432.

[5] D.R.Artusa et al., Searching for neutrinoless double-beta decay of ${ }^{130}$ Te with CUORE, arXiv:1402.6072.

[6] C. P. Aguirre et al., Initial performance of the CUORE-0 experiment,arXiv:1402.0922.

[7] M. Clemenza, C. Maiano, L. Pattavina, and E. Previtali, Radon-induced surface contaminations in low background experiments, Eur. Phys. J. C 711805.

[8] C. Bucci et al., Background study and Monte Carlo simulations for large-mass bolometers, Eur. Phys. J. A 41 (2) 155.

[9] S. Pirro et al., Scintillating double-beta-decay bolometers, Phys. At. Nucl. 69 (12) 2109.

[10] J. W. Beeman et al., Characterization of bolometric light detectors for rare event searches, JINST 8 $\mathrm{P} 07021$.

[11] G. Piperno, S. Pirro, M. Vignati, Optimizing the energy threshold of light detectors coupled to luminescent bolometers, JINST 6 P10005.

[12] J. W. Beeman et al., Performances of a large mass ZnSe bolometer to search for rare events, JINST $\mathbf{8}$ P05021.

[13] J. W. Beeman et al., Performances of a large mass $\mathrm{ZnMoO}_{4}$ scintillating bolometer for a next generation OvDBD experiment, Eur. Phys. J. C 72:2142. 